\title{
EFFECT OF PEDESTRIANS UN-SIGNALIZED MID-BLOCK CROSSING ON VEHICULAR SPEED
}

\author{
B Raghuram Kadali ${ }^{1}$, Tadi Chiranjeevi ${ }^{2}$, Rankireddy Rajesh ${ }^{3}$ \\ 1,2,3 Bapatla Engineering College, Bapatla 522 101, India \\ Received 8 September 2014; accepted 18 February 2015
}

\begin{abstract}
Pedestrian is one of the important component in urban transportation system and also vulnerable at un-protected mid-block locations under mixed traffic conditions. At unprotected mid-block locations, some of the vehicles may yield to pedestrians who are already at crosswalk location. However, some of the pedestrians are using forced gaps to cross the road. Hence, while pedestrians use the mid-block crosswalk with forced gaps, which decreases the vehicular flow characteristics. The pedestrian sidewalks do not show a direct effect on the vehicular flow characteristics when the pedestrian have pleasant walking facilities. The present study has analyzed the effect of pedestrian crossing on the characteristics of vehicular flow at mid-block location under mixed traffic conditions. The results indicate that the pedestrian forced gap condition has significant effect on vehicular characteristics. The study results may be useful for decreasing the travel time for vehicular drivers by controlling usage of pedestrian forced gaps.
\end{abstract}

Keywords: pedestrian, sidewalk, mid-block, cross-flow, vehicular characteristics.

\section{Introduction}

The un-protected mid-block location is one of the important components in the urban transportation system for pedestrian activities under mixed traffic conditions especially in countries like India. The number of such un-protected mid-block pedestrian road crossing activities has been increasing in Indian context and growth of these activities may also result in pedestrian accidents. The increase in un-protected midblock pedestrian road crossings has been significant effect on vehicular characteristics such as an increase in travel times and decrease in vehicle speed. At signalized midblock and intersection, there is the complete right-of-way to pedestrians and vehicles as it results decrease in pedestrian and vehicle conflicts as well as severity of conflicts.

There are numerous studies which deal with the pedestrian road crossing behaviour at intersection and mid-block locations. The importance of these crossing studies is related to the evaluation of pedestrian facilities, traffic control features and road safety treatments by means of before and after crossing studies on pedestrians' behaviour as well as safety. Pedestrians need to cross the road at some location during the course of travel and crosswalks are important for pedestrians to cross the road. The crosswalk locations should provide safe and comfortable movement (Persson, 1988). In general, there are two

\footnotetext{
${ }^{1}$ Corresponding author: brkadali@gmail.com
} 
types of crossings i.e. at-grade and gradeseparated. If the pedestrians are completely segregated (grade-separated) with vehicular traffic, then there is no effect of pedestrian crossings on vehicular flow characteristics. The grade separated facilities are provided exclusively based on the vehicle as well as pedestrian traffic intensity. If, such grade separated crosswalks are too apart from each other, then pedestrians either change their road crossing choice according to their destination which will result in more travel time or pedestrian will use forced gaps to cross the roads. Also, due to poor construction of grade separated facilities and road side development, pedestrians usually cross the road at unprotected mid-block locations under mixed traffic conditions. However, in mixed traffic condition it is very rare to get adequate vehicular gaps to cross the road. Hence, pedestrians will exhibit non-complaint road crossing behaviour, causing more interference with vehicles. It leads to rigorous change in vehicular flow characteristics such as speed and flow. A number of research studies have been carried out on vehicular flow characteristics on freeways, bottlenecks, merged lanes etc., but studies on effect of pedestrian crossing on vehicular flow characteristics are very few.

In this context, the objective of present study is to investigate the effect of pedestrian crossing on vehicular speed. More precisely, this research aims to study the vehicular flow characteristics with and without pedestrian crossings along the same roadway section with same geometry properties. The paper is structured as follows: Section 1 gives the brief introduction of the pedestrian mid-block crossing under mixed traffic conditions. Section 2 describes the literature review. Survey location and methodology is presented in Section 3. Section 4 presents the results. Brief discussions of results are presented in Section 5 and conclusions are presented in Section 6.

\section{Literature Review}

The designing of pedestrian crossing facilities at proper location is a complex problem under mixed traffic conditions in countries like India. The choice of a particular type of pedestrian crossing facilities (at grade or grade separated) influences the safety of pedestrian and results in change of vehicular flow characteristics. It is very important to avoid the sudden change of vehicular flow characteristics caused by unexpected pedestrian crossings by improving typical crossing locations usually by implementing refuge median islands or signalized crossings or complete segregation (grade separated) by considerations of both vehicle as well as pedestrian volume. In this line, Bak and Kiec (2012) studied the influence of mid-block pedestrian crossings on roadway capacity by the simulation model. The results indicate that the vehicular driver willingness to give a right of way to pedestrians on urban roads results in decrease in capacity reduction and increase in delays and it is also observed that there is significant reduction in roadway capacity at zebra crossing locations. Schroeder et al. (2012) found that effect of pedestrian non-complaint behaviour on vehicular capacity at the multilane roundabout as a function of the driver yields behaviour. Duran and Cheu (2012) studied the effect of crosswalk location as well as pedestrian volume on roundabout capacity by the simulation model. From the results, they concluded that if the crosswalk is placed further upstream from the yield line then the entry capacity of roundabout approach increases. But, there is no significant change in the entry capacity when the crosswalk 
is beyond three car-length upstream from the yield line. Silva et al. (2013) studied the effect of crosswalk location on roundabout performance, it was considered with vehicular flow and travel times by the simulation model. The results proved that there is a significant influence of the pedestrian crossing in terms of average travel time and for high vehicle traffic. Ashalatha et al. (2013) studied the effect of bus stops on capacity reduction of urban roads under mixed traffic conditions. From the results, they concluded that bus bays and curb side bus stops can reduce the capacity of urban roadways by $8.1 \%$ and $25.6 \%$ respectively. Chandra et al. (2014) studied the effect of pedestrian cross flow on capacity of urban arterials in mixed traffic condition. From the results, they concluded that pedestrian volume of 100 ped/hour crossing the road will reduce its capacity by 3.52 percent.

Farouki and Nixon (1976) studied the effect of the carriageway width on speed of cars in the special case of free flow conditions in sub-urban roads. From the results, it was found that the mean free speed of cars in suburban area increases linearly with increase in the carriageway width over a certain range of width (5.2m to $11.3 \mathrm{~m})$. Yagar and Van Aerde (1983) found that vehicular traffic speed changes exponentially with change in lane width. Raymond and Knoblauch (2000) studied the effect of crosswalk markings on vehicle speed. From the results, it was found that drivers slightly reduce vehicle speed by yielding to the pedestrians. Hakkert et al. (2002) evaluated the effect of the pedestrian crosswalk warning system on vehicle speed by means of embedded flash lights in pavement. The results inferred that vehicle speed will reduce by 2 to 5 $\mathrm{kmph}$ due to the yielding to pedestrians. Some authors addressed the characteristics of vehicles and pedestrians on different crossing conditions by studying the three conditions of the pedestrian crossing, including crossing freely, crossing at nonsignalized crosswalk, and crossing at the signalized crosswalk. From the results, they concluded that selecting appropriate crossing mode for pedestrians can effectively decrease the vehicle delay, especially when the heavy pedestrian flow exists (Shumin and Yulong, 2007).

The yielding behavior is affected by various aspects of the roadway and driving environment, including vehicle dynamics, pedestrian's behavior, roadway function and design. The driver yield behavior is rarely observed (those pedestrian waiting at curb location) at un-signalized intersection under mixed traffic conditions. The non-complaint behavior of pedestrian and non-driver yield behaviour the interaction between pedestrian-vehicle increases at un-signalized mid-block crosswalk locations. Dulaski and Liu (2013) studied the interaction between the pedestrian and vehicular driver at un-signalized mid-block locations when pedestrian is waiting at curb and stepping off the curb. From the results, it was concluded that, the driver yield behaviour is more when the pedestrian steps off from the curb and it is more during morning peak hours. Safety at mid-block crosswalks depends on the ability of drivers and pedestrians to recognize potential conflicts. Some of the researchers explored pedestrian safety at mid-block crosswalk location and they concluded that pedestrian safety is governed by driver yield behaviour (Brumfield et al., 2013) and some researchers have carried pedestrian road crossing behaviour comparative study between signalized and un-signalized midblock locations (Khatoon et al., 2013). But, there is trade-off between pedestrian safety 
and vehicular flow characteristics (speed, vehicular flow etc.) at un-protected midblock locations due to non-complaint road crossing behaviour of pedestrian. Improper modeling and designing of pedestrian facilities have adverse effects on vehicular flow characteristics and safety of pedestrian. Also the type of regulation (signalized or partial controlled) and road geometry play a key role in vehicular flow characteristics (Brumfield et al., 2013). Ottomanelli et al. (2012) has developed pedestrian vehicle interaction simulation based model and it is useful to evaluate the pedestrian safety as well as performance of the vehicular flow. In the urban transportation system at some locations (school zone and residential areas) the effect of vehicular traffic is reduced by implementing raised pedestrian crosswalks. Some research studies were carried on effect of raised pedestrian crosswalks on urban vehicular traffic speed (Mohammadipour et al., 2012). However, the improper midblock crosswalk location was deliberates the pedestrian crossing behaviour. Moreover this crossing behaviour leaves a deleterious impact on traffic stream. Few research studies address the effect of pedestrian crosswalk on capacity of urban arterials at unprotected mid-block locations under mixed traffic condition. However, very few studies have employed effect of pedestrian crossing on vehicle speed at unprotected mid-block locations under mixed traffic condition. In this background, the objective of the study is to find out the vehicular speed with considerations of vehicle flow characteristics at two different locations with and without pedestrian crossings.

\section{Methodology}

\subsection{Site Selection}

A zebra cross marked location was selected to allow for a minimum pedestrian cross flow at mid-block location to study the effect of pedestrian crossings on vehicular flow. The selected second location is approximately $300 \mathrm{~m}$ away from the previous location on the same roadway corridor with same geometry (mid-block location) and there was a full barrier to prevent the pedestrian crossings for the stable vehicular flow condition. The photographs of the two locations are shown in Fig. 1.

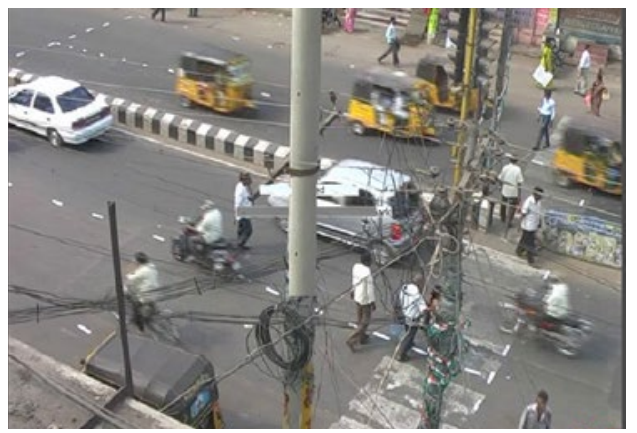

Location (1): Pedestrian zebra cross mid-block

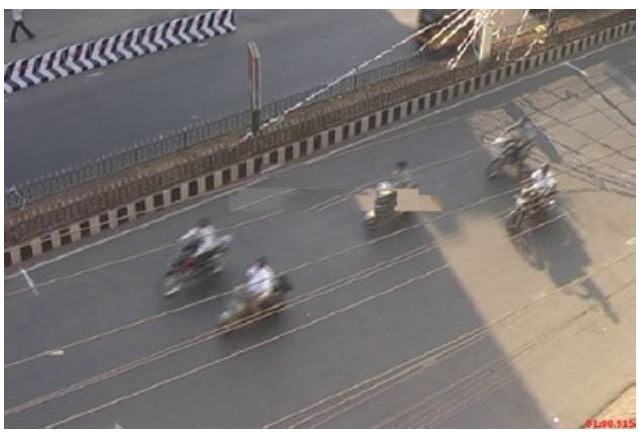

Location (2): Non-pedestrian crossing mid-block 


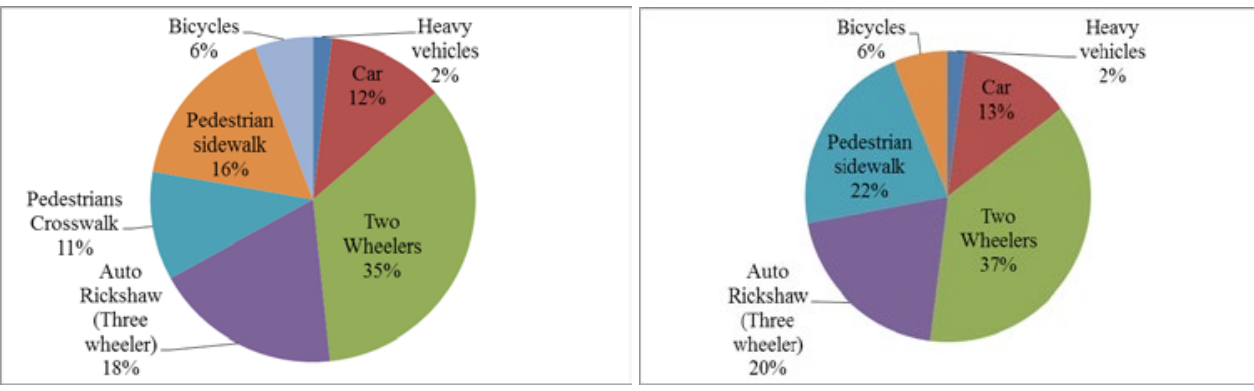

Location (1): Pedestrian zebra cross at mid-block Location (2): Non-pedestrian crossing mid-block

Fig. 1.

Study Locations and Traffic Composition (1) Vehicular Flow Interrupted by Pedestrian Crossing (2)

Uninterrupted Vehicular Flow Condition

\subsection{Data Collection}

Videotaping survey was conducted at both locations during a normal weather working day condition in Guntur, Andhra Pradesh, India. The survey was conducted during two peak flow conditions morning (8:00-10:00 $\mathrm{AM}$ ) and evening (4:00-6:00 PM). The video camera was located on top of a building. The total road section was divided into 20 $\mathrm{m}$ sections of white cello tape to find out the speed of the vehicle and also vehicular flow values. The video was played in Corel studio software in order to stop and view the time frame and data was extracted at an accuracy of 1 in $33 \mathrm{sec}$. From each time step ( $5 \mathrm{~min})$, data were collected which includes pedestrian and traffic characteristics. In particular, to study the individual vehicular effect by pedestrian crossing, data was collected every $5 \mathrm{~min}$ and it is approximated to hourly traffic in order to get the each hour traffic flow characteristics. The collected data include a number of pedestrians, type of vehicle, vehicular flow, and vehicle speed.

\subsection{Model Formulation}

Explanations of traffic flow theory at a point are based upon the three quantities speed $(v)$, flow $(q)$ and density $(k)$ usually considered in traffic flow modeling. Analysis of their definitions leads in the case of homogeneous traffic directly to the formula that has become known as the fundamental equation (Greenshields, 1935). The fundamental equation as follows:

$q=k^{*} v$

Where $q$ is flow, measured in number of vehicles per unit of time, $k$ is density, measured in number of vehicles per length of the road, and $v$ is the space-mean speed (Lighthill and Whitham, 1955), measured in length per unit of time. This fundamental equation can be accompanied by the adoption of a model relationship to describe the association between speed and density as given in Eq. (2). 
$v=f(k)$

Traffic flow can be analyzed effectively based on these relationships ((Lighthill and Whitham, 1955).

When Eq. (2) is populated with an explicit form, any one of the three variables (speed $v$, flow $q$, and density $k$ ) can be used to calculate values for the other two, subject to the ambiguity between free-flow and congested flow values of $q$.

Complex models of traffic flow have been developed that use Eq. (2) as an equilibrium relationship for steady-state flows, towards which traffic speed will relax over time and the same relationships are also used for the unsteady state of flow condition in order to check the vehicular flow.

\section{Results}

\subsection{Observations of Field Study}

The descriptive statistics of the collected speed of the each category of the mode was presented in the Table 1. From the field survey, it is observed that there is significant difference between speeds of the vehicles at selected locations (with and without pedestrian crossing). The hypothesis test was conducted, (the null hypothesis $\mathrm{H}_{0}$ : there is no significant difference between with and without pedestrian crossings on vehicular speeds and alternative hypothesis $\mathrm{H}_{\mathrm{a}}$ : there is significant difference) the null hypothesis rejected from the ANOVA test and corresponding p-value 0.000 and F-value is 27.244. The ANOVA test indicates that there is significant difference means of with and without pedestrian crossing on vehicular speeds.

\section{Table 1}

Vehicular Flow Characteristics Relationships with Pedestrian and without Pedestrian Crossing Conditions

\begin{tabular}{|c|c|c|c|c|c|}
\hline \multirow[b]{2}{*}{ Location } & \multirow[b]{2}{*}{ Type of variable } & \multicolumn{4}{|c|}{ Speed in Kmph } \\
\hline & & Minimum & Maximum & Mean & $\begin{array}{l}\text { Standard } \\
\text { deviation }\end{array}$ \\
\hline \multirow{5}{*}{$\begin{array}{l}\text { Location } 1 \\
\text { (Mid-block } \\
\text { with pedestrian } \\
\text { crossings) }\end{array}$} & All vehicles & 8.6 & 35.86 & 21.04 & 6.85 \\
\hline & Car & 11.4 & 28.96 & 21.4 & 8.84 \\
\hline & Two wheeler & 25.22 & 42.24 & 28.24 & 7.65 \\
\hline & Auto Rickshaw (Three wheeler) & 8.24 & 24.04 & 14.22 & 9.64 \\
\hline & Heavy vehicles & 8.86 & 21.44 & 17.04 & 5.14 \\
\hline \multirow{5}{*}{$\begin{array}{l}\text { Location } \\
2 \text { (Non- } \\
\text { pedestrian } \\
\text { crossing mid- } \\
\text { block) }\end{array}$} & All vehicles & 18.98 & 65.24 & 28.75 & 16.02 \\
\hline & Car & 21.24 & 54.86 & 32.44 & 10.46 \\
\hline & Two wheeler & 26.68 & 64.24 & 33.68 & 9.88 \\
\hline & Auto Rickshaw (Three wheeler) & 17.68 & 36.67 & 21.02 & 8.88 \\
\hline & Heavy vehicles & 14.86 & 32.64 & 19.44 & 7.54 \\
\hline
\end{tabular}




\subsection{Vehicular Flow Characteristics with and without Pedestrian Crossing}

The data such as vehicular flow, speed and density were computed at pedestrian crossing and pedestrian crossing restricted location. In order to study the effect of pedestrian crossing on vehicular flow characteristics the total combined traffic was considered and relationship were plotted between speed and density, speed and flow, flow and density and are presented in Fig. 2. The scattered plot of data points recommended a straightline relation between vehicle speed and density; quadratic relationship between vehicular speed and flow, and vehicular flow and density. In order to study the effect of pedestrian crossing on individual vehicle; individual vehicular flow, speed and density (each type of vehicle separately treated) were also measured from the field data and relationships were also developed. From these individual vehicular characteristics, the driver's yield behaviour at pedestrian crossings can be studied. If the particular vehicular flow characteristics change drastically, it implies that vehicular drivers give more space to the crossing pedestrian. The relationships were developed for combined data (see in Table 2 and Fig. 2) and also for individual mode; the calculated relationships from the analysis of data at two study locations, are presented in Table 2. The correlation coefficient $\mathrm{R}^{2}$ varies from 0.11 to 0.94 at various conditions.

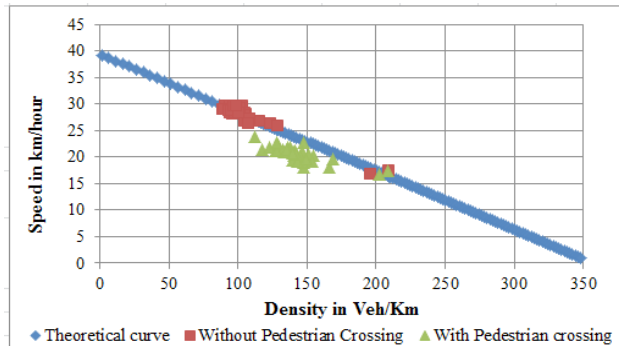

- Theoretical curve $\quad$ Without Pedestrian Crossing $\Delta$ With Pedestrian crossing

Fig. 2 (a)

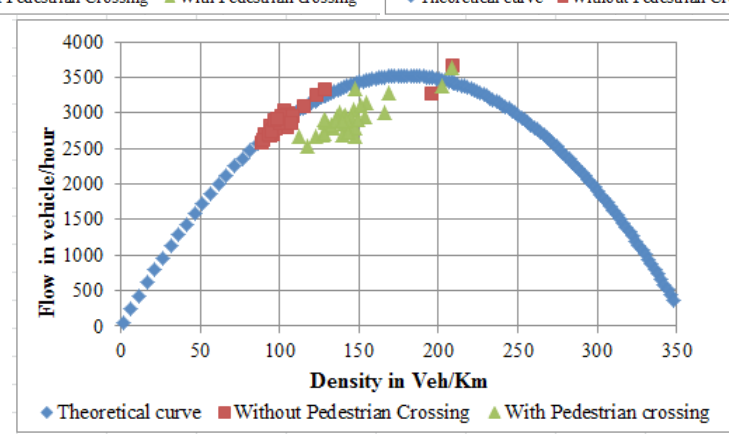

Fig. 2(c)

Fig. 2.

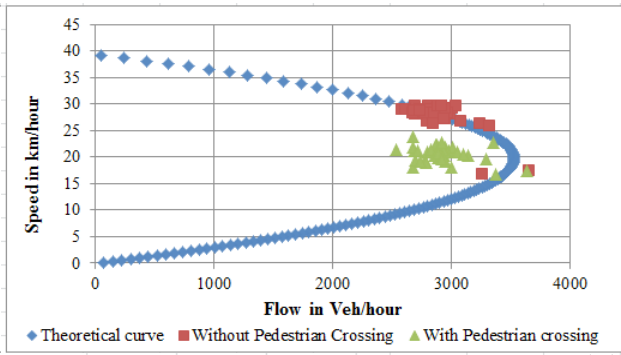

Fig. 2(b)

Traffic Flow Fundamental Diagrams with and without Pedestrian Crossing: (a) Speed and Density Relationship, (b) Speed and Flow Relationship, (c) Flow and Density Relationship 
Table 2

Vehicular Flow Characteristics Relationships with Pedestrian and without Pedestrian Crossing Conditions

\begin{tabular}{|c|c|c|c|c|c|}
\hline Location & $\begin{array}{l}\text { Traffic } \\
\text { Type }\end{array}$ & $\begin{array}{l}\text { Crossing } \\
\text { Condition }\end{array}$ & Relation & Model equation & $\mathbf{R}^{2}$ value \\
\hline \multirow{3}{*}{1} & \multirow{3}{*}{ All vehicles } & \multirow{3}{*}{ With pedestrian } & Speed-density & $\mathrm{v}=29.91-0.065 * \mathrm{k}$ & 0.647 \\
\hline & & & Flow-speed & $\mathrm{q}=\mathrm{v}(29.91-\mathrm{v}) / 0.065$ & 0.212 \\
\hline & & & Flow-density & $\mathrm{q}=\mathrm{k}(29.91-0.065 * \mathrm{k})$ & 0.676 \\
\hline \multirow{3}{*}{1} & \multirow{3}{*}{ Car } & \multirow{3}{*}{ With pedestrian } & Speed-density & $\mathrm{v}=21.83-0.209 * \mathrm{k}$ & 0.404 \\
\hline & & & Flow-speed & $\mathrm{q}=\mathrm{v}(21.83-\mathrm{v}) / 0.209$ & 0.38 \\
\hline & & & Flow-density & $\mathrm{q}=\mathrm{k}(21.83-0.209 * \mathrm{k})$ & 0.618 \\
\hline \multirow{3}{*}{1} & \multirow{3}{*}{$\begin{array}{l}\text { Two } \\
\text { wheeler }\end{array}$} & \multirow{3}{*}{ With pedestrian } & Speed-density & $\mathrm{v}=40.10-0.205^{*} \mathrm{k}$ & 0.645 \\
\hline & & & Flow-speed & $\mathrm{q}=\mathrm{v}(40.10-\mathrm{v}) / 0.205$ & 0.201 \\
\hline & & & Flow-density & $\mathrm{q}=\mathrm{k}\left(40.10-0.205^{*} \mathrm{k}\right)$ & 0.256 \\
\hline \multirow{3}{*}{1} & \multirow{3}{*}{$\begin{array}{l}\text { Auto } \\
\text { Rickshaw } \\
\text { (Three } \\
\text { wheeler) }\end{array}$} & \multirow{3}{*}{ With pedestrian } & Speed-density & $\mathrm{v}=27.47-0.178 * \mathrm{k}$ & 0.415 \\
\hline & & & Flow-speed & $\mathrm{q}=\mathrm{v}(27.47-\mathrm{v}) / 0.178$ & 0.12 \\
\hline & & & Flow-density & $\mathrm{q}=\mathrm{k}\left(27.47-0.178^{*} \mathrm{k}\right)$ & 0.776 \\
\hline \multirow{3}{*}{2} & \multirow{3}{*}{ All vehicles } & \multirow{3}{*}{$\begin{array}{l}\text { Without } \\
\text { pedestrian }\end{array}$} & Speed-density & $\mathrm{v}=39.91-0.101 * \mathrm{k}$ & 0.938 \\
\hline & & & Flow-speed & $\mathrm{q}=\mathrm{v}(39.91-\mathrm{v}) / 0.101$ & 0.565 \\
\hline & & & Flow-density & $\mathrm{q}=\mathrm{k}\left(39.91-0.101^{*} \mathrm{k}\right)$ & 0.711 \\
\hline \multirow{3}{*}{2} & \multirow{3}{*}{ Car } & \multirow{3}{*}{$\begin{array}{l}\text { Without } \\
\text { pedestrian }\end{array}$} & Speed-density & $\mathrm{v}=29.89-0.264^{*} \mathrm{k}$ & 0.233 \\
\hline & & & Flow-speed & $\mathrm{q}=\mathrm{v}(29.89-\mathrm{v}) / 0.264$ & 0.121 \\
\hline & & & Flow-density & $\mathrm{q}=\mathrm{k}\left(29.89-0.264^{*} \mathrm{k}\right)$ & 0.256 \\
\hline \multirow{3}{*}{2} & \multirow{3}{*}{$\begin{array}{l}\text { Two } \\
\text { wheeler }\end{array}$} & \multirow{3}{*}{$\begin{array}{l}\text { Without } \\
\text { pedestrian }\end{array}$} & Speed-density & $\mathrm{v}=42.43-0.188^{*} \mathrm{k}$ & 0.556 \\
\hline & & & Flow-speed & $\mathrm{q}=\mathrm{v}(42.43-\mathrm{v}) / 0.188$ & 0.112 \\
\hline & & & Flow-density & $\mathrm{q}=\mathrm{k}\left(42.43-0.188^{*} \mathrm{k}\right)$ & 0.256 \\
\hline \multirow{3}{*}{2} & \multirow{3}{*}{$\begin{array}{l}\text { Auto } \\
\text { Rickshaw } \\
\text { (Three } \\
\text { wheeler) }\end{array}$} & \multirow{3}{*}{$\begin{array}{l}\text { Without } \\
\text { pedestrian }\end{array}$} & Speed-density & $\mathrm{v}=34.92-0.293 * \mathrm{k}$ & 0.769 \\
\hline & & & Flow-speed & $\mathrm{q}=\mathrm{v}(34.92-\mathrm{v}) / 0.293$ & 0.110 \\
\hline & & & Flow-density & $\mathrm{q}=\mathrm{k}(34.92-0.293 * \mathrm{k})$ & 0.252 \\
\hline
\end{tabular}

\section{Discussion}

The mathematical relationships were developed between speed, density and flow in the present study. The theoretical vehicular speed was found as $30 \mathrm{kmph}$ at pedestrian crossing locations and $40 \mathrm{kmph}$ at restricted pedestrian crossing location (See in Table 2, if the density $(k)$ equal to zero for all the vehicles case). From the field survey, it is also observed that there is a reduction in speed $(7.7 \mathrm{kmph})$ at pedestrian crossing location as compared to the pedestrian crossing restricted locations. The speed represented here are average speed of all the vehicles, including two wheeler, car and auto rickshaw (three-wheeler). The interaction between pedestrian and vehicles will be more at un-signalized pedestrian crosswalk locations and the change in vehicular flow characteristics increase with an increase in non-complaint behavior of pedestrian as well as multiple stage of road crossing behavior of pedestrian. From the field survey, it is observed that, the higher jaywalking or higher multiple stage of road crossing behavior (forced gaps), parked vehicles and waiting pedestrians for bus or 
auto further reduction in roadway width results in an increase in interaction between vehicles and pedestrian. It leads to further reduction in vehicular speed as well as other characteristics (flow etc.). The theoretical capacities were observed as 3676 vehicles/ hour and 2486 vehicles/hour without pedestrian crossing and with pedestrian crossings respectively. The fundamental diagrams of vehicular characteristics (all vehicles) with and without pedestrian crossings are shown in the Fig. 2. It shows that there is a clear drop of the average vehicle speed, increasing density and reduced in vehicular flow with pedestrian crossing.

\subsection{Variation in Vehicle Flow Characteristics with Type of Vehicle Considering Effect of Pedestrian Crossings}

The effects of pedestrian crossing on individual vehicles were studied by considering the variation of individual vehicle flow characteristics. From the field survey, it is observed that vehicles such as car, two wheeler and auto rickshaw (three wheeler) were more compared to heavy vehicles (bus and trucks) at the selected site. So, the individual variation was carried for only car, two wheeler and auto rickshaw. From this study, the individual driver behaviour with effect of pedestrian crossing can be observed. The variation of car vehicle flow characteristics is shown in Fig. 3. In particular, the average car speed (theoretical speed which indicates that $k=0$ from the Table 2) was observed at pedestrian crossing is $21.83 \mathrm{kmph}$ and at pedestrian crossing restricted location observed as $\mathbf{2 9 . 8 9}$ $\mathrm{kmph}$. Due to interference the car drivers are more affected due to pedestrian crossings and interaction between pedestrian-car is also high. The variation of two wheeler characteristics with and without pedestrian crossing is shown in Fig. 4. The two wheeler speed was found as $42.43 \mathrm{kmph}$ without pedestrian crossing and $40.1 \mathrm{kmph}$ with pedestrian crossing (theoretical speed which indicates that $k=0$ for two-wheelers from the Table 2). It is clearly indicated that there is no significant effect of pedestrian crossing on two wheelers when compared to the car. It is also observed from the field survey, the two wheeler drivers change their vehicular path instead of change in speed to yield to pedestrians at crosswalk locations. From the Fig. 3(b) and Fig. 4(b) of vehicle speed and flow relationship, the theoretical capacity value is higher for the case of two wheelers when compared to the cars because there is no change in two wheeler speed. It is the indirect indication of the effect of pedestrian crossing on two wheelers. 

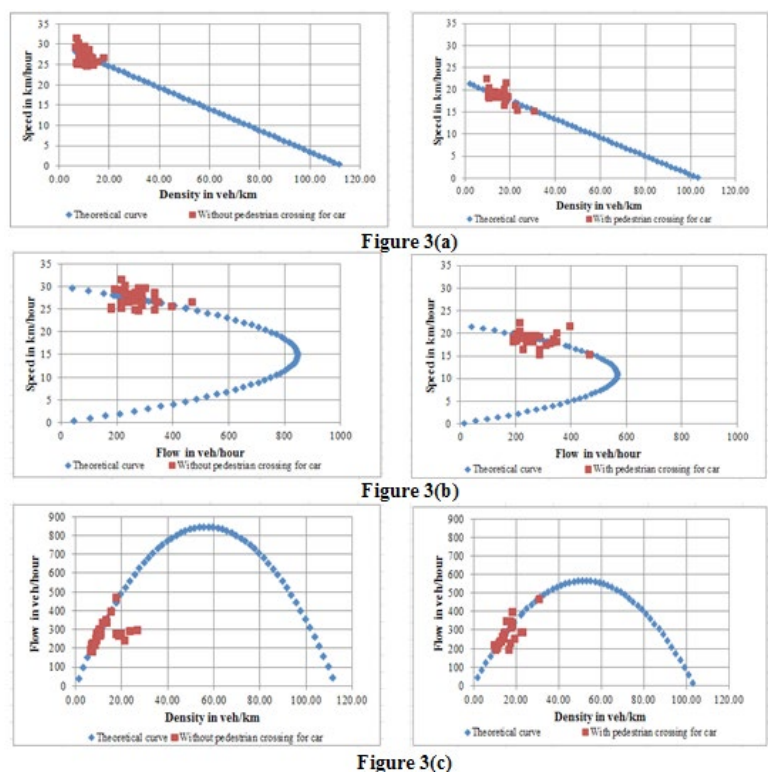

Fig. 3.

Traffic Flow Fundamental Diagrams with and without Pedestrian Crossing for Car: (a) Speed and Density Relationship, (b) Speed and Flow Relationship, (c) Flow and Density Relationship

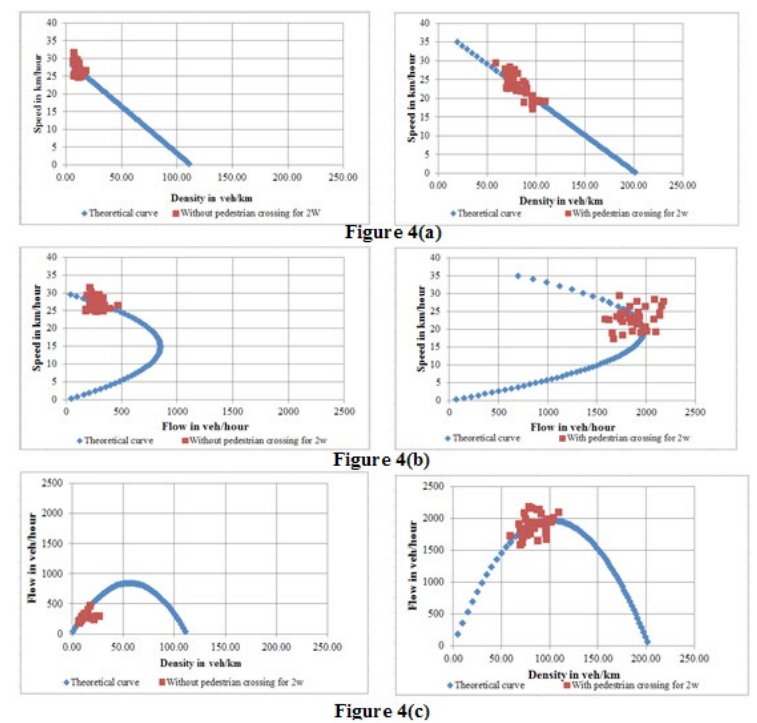

Fig. 4.

Traffic Flow Fundamental Diagrams with and without Pedestrian Crossing for Two Wheeler: (a) Speed and Density Relationship, (b) Speed and Flow Relationship, (c) Flow and Density Relationship 
The variation of auto rickshaw characteristics with and without pedestrian crossings is shown in Fig. 5. The average speed of auto rickshaw was observed as $34.92 \mathrm{kmph}$ when the pedestrian crossing restricted and 27.47 $\mathrm{kmph}$ with pedestrian crossing (theoretical speed which indicates that $k=0$ for auto rickshaw (three wheeler) from the Table 2). There is a significant change in auto rickshaw speed with effect of pedestrian crossing. From these results, it inference that car and auto rickshaw has significantly changed their vehicular speed while yielding to pedestrians who are already in crosswalk location under mixed traffic conditions. In case of two wheelers, there is possibility of change in their vehicular paths to yield to pedestrians when they are in crosswalk location. However, the overall flow characteristics of car and auto rickshaw were changed drastically when compared to the two wheelers. Some research studies were carried to study the effect of pedestrian crossings on vehicular flow by converting all the vehicles into single passenger car unit (PCU) value (Chandra et al., 2014). But, in this study, the analysis was carried with individual vehicles in order to quantify the effect of pedestrian crossing on each individual vehicle. Furthermore, there is an increase in pedestrian cross flow leading to further reductions in vehicle speeds, because the pedestrian platoon has higher chance to cross the road when compared to individual pedestrians.
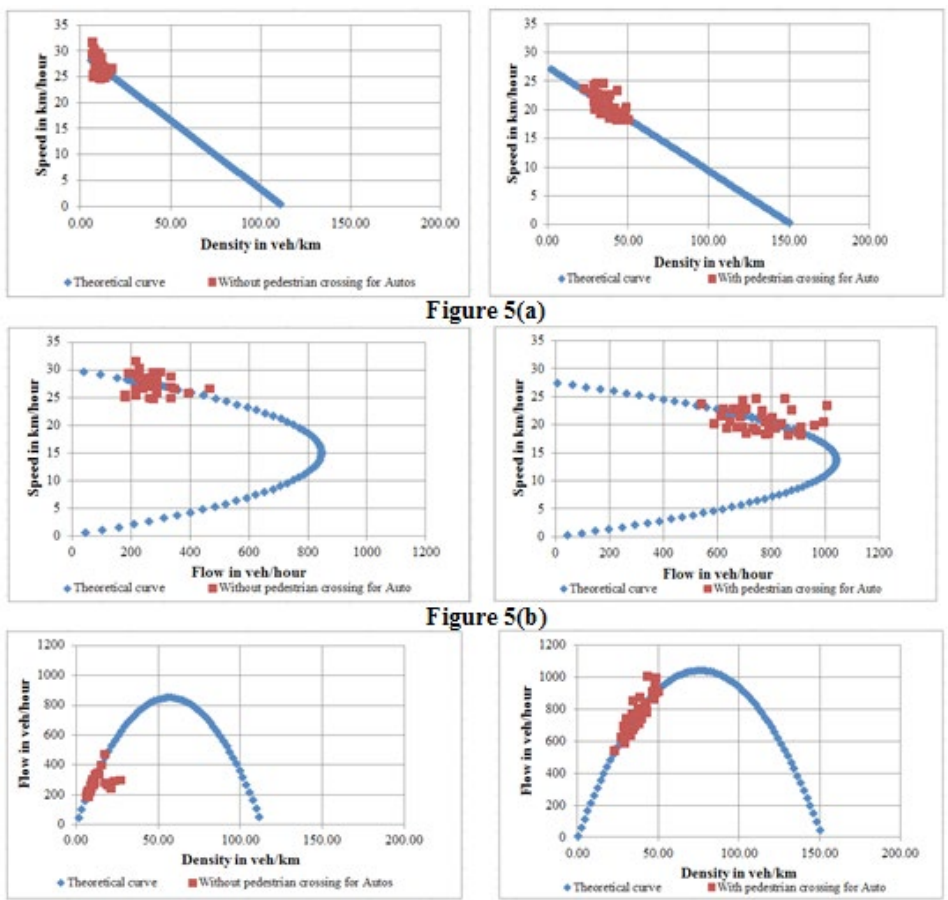

Figure 5(b)

\section{Figure 5(c)}

Fig. 5.

Traffic Flow Fundamental Diagrams with and without Pedestrian Crossing for Auto Rickshaw: (a) Speed and Density Relationship, (b) Speed and Flow Relationship, (c) Flow and Density Relationship 
Early research studies show that the effect of reduction carriageway width by bus stops on urban capacity (Ashalatha et al., 2013). In this study, it is found that there is significant effect of parked vehicle or improper bus stop locations on vehicular flow characteristics. Perhaps, the improper bus stop locations leads to pedestrian cross the road at any point of mid-block locations and further the non-complaint behavior of pedestrian increase with increase in irregularity of stopping vehicles for passenger boarding and alighting (this is not only for the public transportation like bus, it is also true with Para-transportation system such as auto rickshaw in countries like India). Further, these vehicles (auto rickshaw) more flexibility in stops at any point of location also leads to cross the pedestrian at different point of mid-block with or without crosswalks. The present study highlights the change in vehicular speeds with respect to pedestrian crossings and it found that there is significantly reduction in vehicle speed.

\section{Conclusions}

The vehicular flow characteristics were studied at un-signalized mid-block pedestrian crossing and pedestrian restricted crossing in Guntur, India. The vehicular speeds were implicitly affected with pedestrian crossing when compared to without pedestrian crossing location under mixed traffic conditions. The theoretical capacity is significantly reduced with pedestrian crossings for car. However, increase in capacity is observed with pedestrian crossings in case of two-wheeler. The underlying fact is the variation of the speed of the car and two-wheeler. In case of auto rickshaw (three wheeler) also there is a significant drop of speed value. From this study, it concluded that the car and auto rickshaw driver yielding to pedestrians in terms of speed is more when compared to the two wheeler drivers. The increase in reduction of vehicle speed significantly affects the travel time of vehicular drivers and it further has influence on the fuel consumption. However, the driver yield behaviour is the tradeoff between pedestrian safety and vehicular flow characteristics. This study clearly indicates that the importance of pedestrian crossing facilities and the barrier effect on the vehicular flow characteristics. The judgment of segregating pedestrians from vehicular traffic should be based on the number of pedestrian accidents, illegal pedestrian crossing and demand of pedestrian as well as vehicular flow. However, this study has some limitations, in this study the effect of pedestrian crossing on the heavy vehicle is not addressed because of less heavy vehicle flow at selected location. This study result is restricted to single location of 4-lane divide roadway characteristics. There is a need to study the other crosswalk locations with varied roadway geometry and crosswalk types. It is also important to address the effect of pedestrian behavioural characteristics (change in pedestrian speed as well as crossing path) on vehicular characteristics under mixed traffic condition. In spite of these limitations, this study result has great inferences for pedestrian crossing facilities for urban planning policies and design practices for controlling the pedestrian crossings as well as proper location for the pedestrian crossing under mixed traffic conditions. 


\section{References}

Ashalatha, R.; Salini, S.; Prakash, N. 2013. Capacity Reduction of Urban Roads Due to Bus Stops. In Proceedings of the 92 nd Transportation Research Board Annual meeting, Washington D.C., 1-10.

Bak, R.; Kiec, M. 2012. Influence of Various Types of Mid-Block Pedestrian Crossings on Urban Street Capacity. In Proceedings of the 91st Transportation Research Board Annual meeting, Washington D.C., 1-12.

Brumfield, R.M.; Pulugurtha, S.S.; Maradapudi, J.M.R.; Miatudila, A.S. 2013. Effect of Road User Distractions on Pedestrian Safety at Mid-block Crosswalks on a College Campus. In Proceedings of the 92nd Transportation Research Board Annual meeting, Washington D.C., 1-12.

Chandra, S.; Rao, G.S.; Dhamaniya, A.Z. 2014. Effect of Pedestrian Cross-Flow on Capacity of Urban Arterials, Indian Highways, 51-58.

Dulaski, D.M.; Liu, Y. 2013. Stepping off the Curb to Increase Drivers' Yielding Behavior at Mid-block Crosswalks. In Proceedings of the 92nd Transportation Research Board Annual meeting, Washington D.C., 1-13.

Duran, C.; Cheu, R.L. 2012. Effect of Crosswalk Location and PedestrianVolume on Entry Capacity of Roundabouts. In Proceedings of the 91st Transportation Research Board Annual meeting, Washington D.C., 1-20.

Farouki, O.T; Nixon, W.J. 1976. The Effect of Width of Sub-Urban Roads on the Mean Free Speeds of Cars, Traffic Engineering Control, 17(12): 518-519.

Greenshields, B.D. 1935. A study of traffic capacity, Highway Research Board Proceedings, 14: 448-477.

Hakkert, A.S; Gitelman, V.; Ben-Shabat, E. 2002. An evaluation of crosswalk warning systems: effects on pedestrian and vehicle behaviour, Transportation Research PartF: Traffic Psychology and Behaviour. DOI: http://dx.doi. org/10.1016/S1369-8478(02)00033-5, 5(4): 275-292.
Khatoon, M.; Tiwari, G.; Chatterjee, N. 2013. Modelling of pedestrian unsafe road crossing behavior: A comparison at a signalized and a non-signalized crosswalk. In Proceedings of the 92nd Transportation Research Board Annual meeting, Washington D.C., 1-12.

Lighthill, M.J.; Whitham, G.B. 1955. On kinematic waves: II. A theory of traffic flow on long crowded roads, Proceedings of the Royal Society. DOI: http://dx.doi. org/10.1098/rspa.1955.0089, 229(1178): 317-345.

Mohammadipour, A.H.; Archilla, A.R.; Papacostas, C.S.; Alavi, S.H. 2012. Raised Pedestrian Crosswalk (RPC) Influence on Speed Reduction. In Proceedings of the 91st Transportation Research Board Annual meeting, Washington D.C., 1-20.

Ottomanelli, M.; Iannucci, G.; Sassanelli, D. 2012. A Simplified Pedestrians-Vehicles Interaction Model at Road Crossings Based on Discrete Events System. In Proceedings of the 91st Transportation Research Board Annual meeting, Washington D.C., 1-12.

Persson, H. 1988. Communication between Pedestrian and Car Drivers. Unpublished report, Lund University, Lund, Sweden.

Raymond, P.D.; Knoblauch, R.L. 2000. The effect of crosswalk marking on vehicle speeds. In Proceedings of the 79th Transportation Research Board Annual meeting, Washington D.C., Paper No. 00-1602.

Schroeder, B.; Rouphail, N.M.; Salamati, K.; Bugg, Z. 2012. Effect of Pedestrian Impedance on Vehicular Capacity at Multilane Roundabouts with Consideration of Crossing Treatments, Transportation Research Record. DOI: http://dx.doi.org/10.3141/231202, 2312: 14-24.

Shumin, F.; Yulong, P. 2007. Analysis of Vehicle Delay on Road Sections on the Condition of Pedestrian crossing, Journal of Transportation Systems Engineering and Information Technology. DOI: http://dx.doi.org/10.1016/S15706672(07)60023-3, 7(3): 73-77. 
Silva, A.B.; Cunha, J.; Relvão, T.; Silva, J.P. 2013. Evaluation of the effect of the pedestrian crossings on roundabout performance using microsimulation. In Proceedings of the 92nd Transportation Research Board Annual meeting, Washington D.C., 1-14.

Yagar, S.; Van Aerde, M. 1983. Geometric and Environmental Effects on Speeds of Two Lane Highways, Transportation Research Part A: General. DOI: http://dx.doi.org/10.1016/0191-2607(83)90094-8, 17(4): 315-325. 